\title{
An Optimized Protocol for First Strand cDNA Synthesis from Laser Capture Microdissected Tissue
}

\author{
Sharon Boylan, Shigeru Honda, Leonard M. Hjelmeland, and James T. Handa \\ Departments of Ophthalmology (SB, SH, LMH, JTH) and Molecular and Cellular Biology (LMH), University of \\ California Davis, Davis, California
}

$L$ aser capture microdissection (LCM) is a novel technique that collects pure populations of morphologically similar cells from histopathologic tissue for subsequent molecular analysis (Emmert-Buck et al, 1996). The development of LCM has challenged the sensitivity of any chosen molecular assay because the amount of material obtained from histopathologic tissue is limited. Our laboratory has been determining the mRNA phenotypes of cells in the retinal pigment epithelium (RPE) in health and age-related macular degeneration (AMD), the most common cause of blindness among the elderly in Western society. In AMD, the RPE undergoes characteristic morphologic derangement in an area $6 \mathrm{~mm}$ in diameter. The limited amount of tissue and the need to obtain pure cell populations to identify accurate gene expression profiles have prompted the development of a protocol that maximizes the recovery of RNA from limited numbers of cells dissected by LCM for reverse transcriptase-polymerase chain reaction (RT-PCR). Since Bosch et al (2000) suggested that RNA availability and the sensitivity of the $\mathrm{RT}$ reaction, not the source of taq polymerase, were the limiting factors in differential display expression profiles from limited amounts of tissue, our investigation focused on maximizing the recovery of RNA and synthesis of first strand cDNA.

Initial experiments using the National Institutes of Health LCM website protocol (http://dir.nichd.nih.gov/lcm/lcm.htm) for RNA extraction (Stratagene Microisolation kit; Stratagene, La Jolla, California) and RT-PCR with either laser captured RPE or ARPE-19 RPE cells, an established RPE cell line with highly differentiated characteristics (Handa et

Received April 5, 2001.

SB and SH contributed equally to this work. No commercial relationships were involved.

This work was supported by National Institutes of Health Grants NIH/ EY00344 (JTH) and NIH/EYO6473 (LMH), University of California Davis Health System Awards (JTH), Manpower Award (JTH), and an unrestricted RPB grant from Research to Prevent Blindness to the Department of Ophthalmology and Nippon Eye Bank Association (SH).

Address reprint requests to: Dr. James T. Handa, Wilmer Eye Institute, 3-109 Jefferson Building, 600 N. Wolfe St., Baltimore, MD 21287.

E-mail: jthanda@jhmi.edu al, 1998), gave inconsistent results when using primers for glyceraldehyde-3-phosphate dehydrogenase $(G A P D H)$, a high copy gene, or plateletderived growth factor (PDGF)-A chain, a low copy gene. This RNA extraction protocol uses alcohol precipitation, which can reduce RNA yield by $50 \%$ (Hengen, 1996). Extraction methods have been developed that avoid alcohol precipitation, where RNA preferentially binds to a silica-gel membrane on a column while contaminants are washed away (Qiagen, 2001). RT-PCR analysis using RNA extracted from a column-based extraction method yielded a 10 -fold increase in sensitivity over the alcohol precipitation protocol, with an improved ability to see GAPDH on an ethidium bromide-stained agarose gel from $10^{5}$ to $10^{4}$ ARPE-19 cells ( $n=3$; data not shown). Therefore, all subsequent experiments used the column-based extraction method.

The activity of two reverse transcriptases (RT) (Superscript II; Gibco BRL, Grand Island, New York) and Sensiscript (Qiagen, Valencia, California), which have been reported to be among the most sensitive, were compared (Bosch et al, 2000). Sensiscript consistently allowed visualization of GAPDH with as few as $10^{3}$ ARPE-19 cells compared with $10^{4}$ cells when using Superscript II ( $n=3$; data not shown). Sensiscript may have an advantage over Superscript II because it has a high priming capacity, which rescues the expression of low abundant RNA species (Bosch et al, 2000). RT can potentially inhibit taq polymerase activity by remaining bound to the newly synthesized first strand cDNA. Although heat inactivates RT activity, it may not release RT from the cDNA. T4 gene 32 protein, a single stranded DNA binding protein and a double stranded DNA helicase (Alberts and Sternglanz, 1977; Morris et al, 1975) that improves sensitivity during PCR amplification (Kreader, 1996; Vahjen and Tebbe, 1994), has also been found to facilitate the release of RT from first strand CDNA and relieve the inhibitory effect on taq (Chandler et al, 1998). Inclusion of T4 gene 32 protein resulted in a remarkable increase in sensitivity, so that GAPDH was identified on an ethidium bromide-stained agarose gel with 100 cells using Superscript II and with as few as 10 ARPE-19 cells with Sensiscript (Fig. 1). 
A

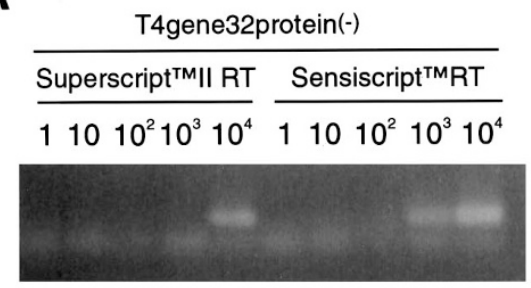

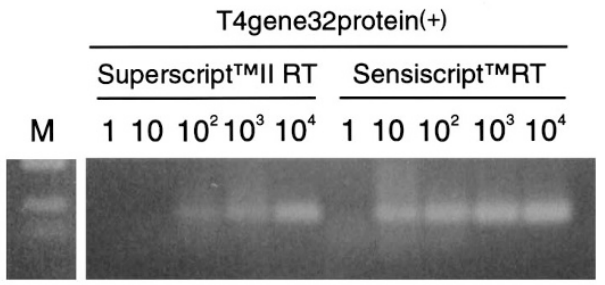

B

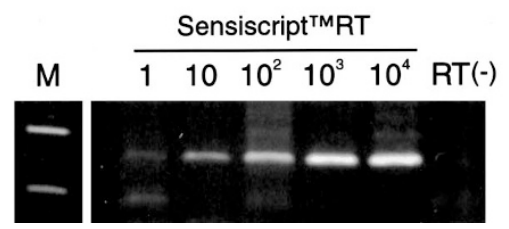

\section{Figure 1.}

A, Ethidium bromide-stained agarose gel of reverse transcriptase-polymerase chain reaction (RT-PCR) product for glyceraldehyde-3-phosphate dehydrogenase (GAPDH, 157 bp) after 40 cycles using the primers and PCR conditions of Tong et al (1997). These primers were selected because they span consecutive exons, which obviates the need for DNase treatment to remove contaminating DNA. RNA was extracted with an RNeasy Minikit (Qiagen, Valencia, California) and first strand CDNA was prepared using Superscript II RNase H- (Gibco BRL, Grand Island, New York) or Sensiscript (Qiagen), according to the manufacturers' recommendations. The reverse transcriptase (RT) reaction was performed in a final volume of $20 \mu$ l with $8 \mu$ l of total RNA and $10 \mu \mathrm{M}$ oligo (dT) primers at $37^{\circ} \mathrm{C}$ for 90 minutes. Some reactions included $1.5 \mu \mathrm{g}$ T4 gene 32 protein (Ambion, Austin, Texas). B, The same reaction seen in the right panel of A, but visualized with an ethidium bromide-stained $10 \%$ polyacrylamide gel, which significantly improves the resolution.

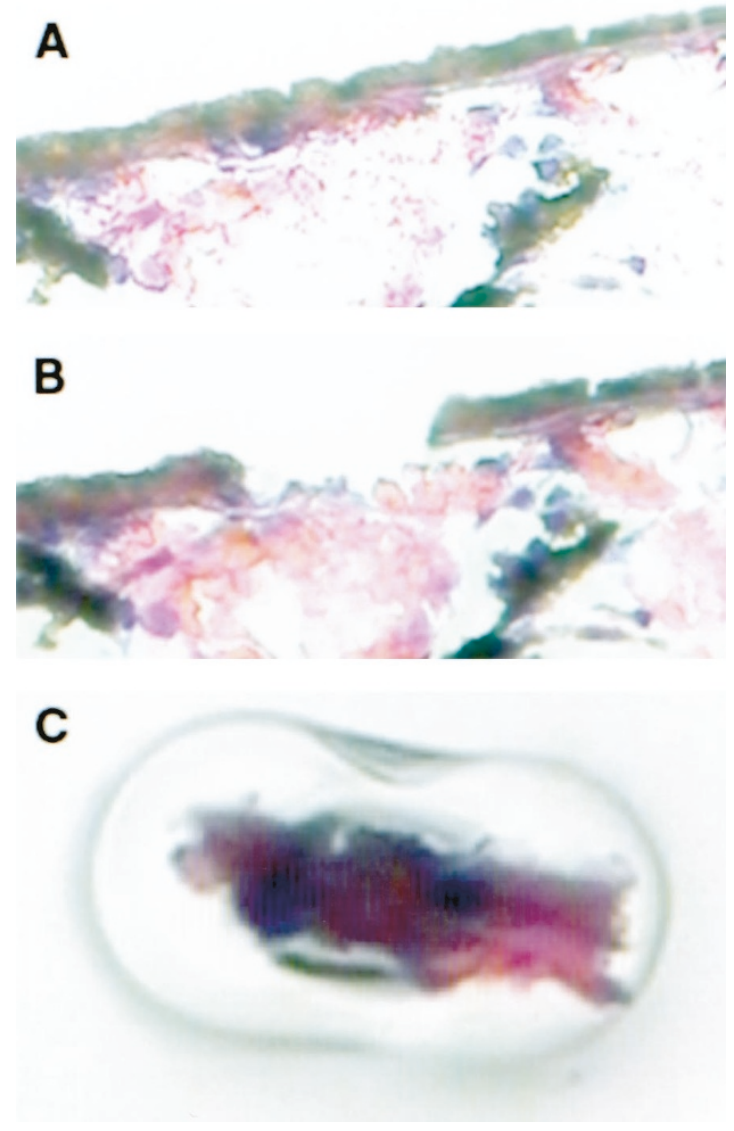

Figure 2.

A globe from a 60-year-old donor was obtained 16 hours after death and cryopreserved without fixative as recommended by Goldsworthy et al (1999). Hematoxylin and eosin stained $10-\mu \mathrm{m}$ sections showing retinal pigment epithelial (RPE) cells before (A) and after (B) laser capture microdissection with a PixCell II (Arcturus Engineering, Mountain View, California). C, Three RPE cells which are adherent to the transfer membrane after dissection are visualized.
The resolution is improved so that GAPDH can be seen from 1 ARPE-19 cell when a polyacrylamide gel is used.

The sensitivity of this protocol was evaluated using laser-captured morphologically normal RPE cells from a 60-year-old donor eye that was cryopreserved without fixative as recommended by Goldsworthy et al (1999) (Fig. 2). With an ethidium bromide-stained polyacrylamide gel, GAPDH was visualized from a

A

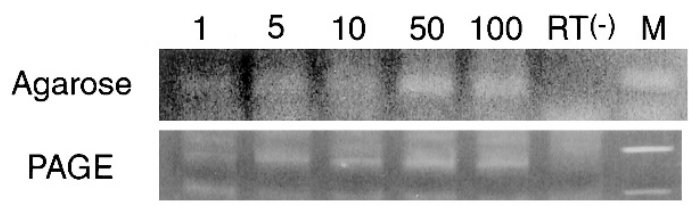

Southern

B

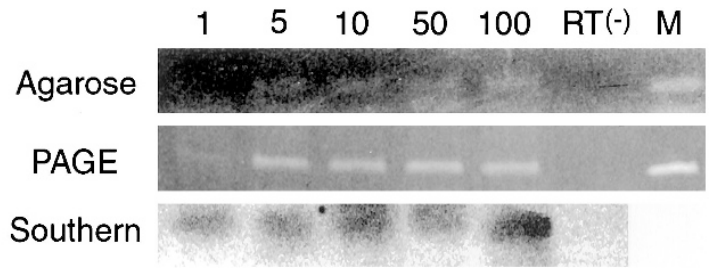

Figure 3.

A, RT-PCR analysis of GAPDH (157 bp) is seen on an ethidium bromidestained agarose gel (top), 10\% polyacrylamide gel (middle), and Southern hybridization using a ${ }^{32} \mathrm{P}$-labeled internal probe with as little as one laser capture microdissection (LCM) RPE cell (bottom). B, RT-PCR analysis of platelet-derived growth factor (PDGF)-A chain (223 bp) using the primers and conditions of Osterlund et al (1996) from LCM RPE cells as visualized by an ethidium bromide-stained agarose gel (top), 10\% polyacrylamide gel (middle), and Southern analysis with as little as one cell (bottom). 
single laser-captured RPE cell. Southern analysis with an internal probe confirmed that the band at $157 \mathrm{bp}$ is likely GAPDH (Fig. 3). The sensitivity of this method was further confirmed by visualizing PDGF-A chain, a low copy gene, from five laser-captured cells using an agarose gel and with one cell on a polyacrylamide gel. Southern analysis with an internal probe also confirmed the band from one laser-captured RPE cell. These results compare favorably with other studies using LCM tissue that required double or nested PCR (Bernsen et al, 1998; Jin et al, 1999). Like the work of Kohda et al (2000), Southern transfer of RT-PCR products and hybridization with an internal probe provided confirmatory evidence of reaction specificity. We find also that Southern hybridization increased our ability to visualize the band of interest, especially with low starting cell numbers. Thus, we find that avoiding RNA extraction, which uses alcohol precipitation, and using Sensiscript RT with T4 gene 32 protein markedly improved the sensitivity of RT-PCR on laser-captured tissue.

\section{References}

Alberts B and Sternglanz R (1977). Recent excitement in the DNA replication problem. Nature 269:655-661.

Bernsen MR, Dijkman HB, de Vries E, Figdor CG, Ruiter DJ, Adema GJ, and van Muijen GN (1998). Identification of multiple mRNA and DNA sequences from small tissue samples isolated by laser-assisted microdissection. Lab Invest 78:1267-1273.

Bosch I, Melichar H, and Pardee AB (2000). Identification of differentially expressed genes from limited amounts of RNA. Nucleic Acids Res 28:E27.

Chandler DP, Wagnon CA, and Bolton H, Jr (1998). Reverse transcriptase (RT) inhibition of PCR at low concentrations of template and its implications for quantitative RT-PCR. Appl Environ Microbiol 64:669-677.

Emmert-Buck MR, Bonner RF, Smith PD, Chuaqui RF, Zhuang Z, Goldstein SR, Weiss RA, and Liotta LA (1996). Laser capture microdissection [see comments]. Science 274: 998-1001.
Goldsworthy SM, Stockton PS, Trempus CS, Foley JF, and Maronpot RR (1999). Effects of fixation on RNA extraction and amplification from laser capture microdissected tissue. Mol Carcinog 25:86-91.

Handa JT, Reiser KM, Matsunaga $\mathrm{H}$, and Hjelmeland LM (1998). The advanced glycation endproduct pentosidine induces the expression of PDGF-B in human retinal pigment epithelial cells. Exp Eye Res 66:411-419.

Hengen PN (1996). Carriers for precipitating nucleic acids. Trends Biochem Sci 21:224-225.

Jin L, Thompson CA, Qian X, Kuecker SJ, Kulig E, and Lloyd RV (1999). Analysis of anterior pituitary hormone mRNA expression in immunophenotypically characterized single cells after laser capture microdissection. Lab Invest 79:511512.

Kohda Y, Murakami H, Moe OW, and Star RA (2000). Analysis of segmental renal gene expression by laser capture microdissection. Kidney Int 57:321-331.

Kreader CA (1996). Relief of amplification inhibition in PCR with bovine serum albumin or T4 gene 32 protein. Appl Environ Microbiol 62:1102-1106.

Morris CF, Sinha NK, and Alberts BM (1975). Reconstruction of bacteriophage T4 DNA replication apparatus from purified components: Rolling circle replication following de novo chain initiation on a single-stranded circular DNA template. Proc Natl Acad Sci USA 72:4800-4804.

Osterlund C, Wramsby H, and Pousette A (1996). Temporal expression of platelet-derived growth factor (PDGF)-A and its receptor in human preimplantation embryos. Mol Hum Reprod 2:507-512.

Qiagen (2001). RNeasy Mini Handbook. Valencia, CA: Qiagen.

Tong D, Schneeberger C, Leodolter S, and Zeillinger R (1997). Quantitative determination of gene expression by competitive reverse transcription-polymerase chain reaction in degraded RNA samples. Anal Biochem 251:173-177.

Vahjen W and Tebbe CC (1994). Enhanced detection of genetically engineered Corynebacterium glutamicum pUN1 in directly extracted DNA from soil, using the T4 gene 32 protein in the polymerase chain reaction. Eur $\mathrm{J}$ Soil Biol 30:93-98. 